\title{
Facemasks and COVID-19 case fatality rate
}

\author{
Zacharias Fögen ${ }^{1}$ \\ ${ }^{1}$ Affiliation not available
}

March 5, 2021

\begin{abstract}
Mask mandates have been a globally used epidemiologic intervention during the ongoing COVID-19 (coronavirus disease 2019) pandemic. Although there is extensive supporting literature on the use of facemask to reduce infection rates, its effect on the individual and course of disease has remained controversial. The purpose of this study was to find if mandatory masking influences the case fatality rate. This study used data on case updates, mask mandates, and demographic status related to the Kansas state, USA. The data were analyzed using a parallelization approach based on county-level data.

The results showed that in Kansas during the summer of 2020, the counties with mask mandate had significantly higher case fatality rates compared to counties without mask mandate, with a risk ratio of 1.85 [1.51-2.10] for death with COVID-19.

Even after adjusting for the number of 'protected persons', i.e., the number of persons who were not infected in the maskmandated group compared to the no-mask group, the risk ratio remained significantly high at 1.52 [1.24-1.72]. By analyzing the excess mortality in Kansas, this study determines that over $95 \%$ of this effect can solely be attributed to COVID-19. The cause of this trend and the possible connection between long-term effects associated with SARS-CoV-2 and facemasks are explained in the theory herein by the 'foegen effect'; i.e., deep reinhalation of pure virions caught in the facemasks as droplets can worsen the prognosis.

This finding suggests that the use of facemasks in COVID-19 pandemic did contribute to an increase in the death toll counterintuitive of its purpose, making mask mandates a highly debatable epidemiologic intervention.
\end{abstract}

\section{Zusammenfassung (German translation of abstract)}

Maskengebote waren eine weltweit eingesetzte epidemiologische Intervention während der laufenden COVID-19-Pandemie (Coronavirus-Krankheit 2019). Obwohl es umfangreiche unterstützende Literatur über die Verwendung von Gesichtsmasken zur Verringerung der Infektionsraten gibt, ist die Wirkung auf das Individuum und den Krankheitsverlauf umstritten geblieben. Das Ziel dieser Studie war es, herauszufinden, ob das obligatorische Maskentragen die Fallsterblichkeitsrate beeinflusst. In dieser Studie wurden Daten zu COVID-19-Fällen, zur Maskenpflicht und zur Sterblichkeit bezogen auf den Bundesstaat Kansas, USA, verwendet. Die Daten wurden mit Hilfe eines Parallelisierungsansatzes auf der Grundlage von Daten auf Bezirksebene analysiert.

Die Ergebnisse zeigten, dass in Kansas im Sommer 2020 die Bezirke mit Maskenmandat eine signifikant höhere Todesfallrate im Vergleich zu Bezirken ohne Maskenmandat hatten, mit einem relativen Risiko von 1,85 [1,51-2,10] für den Tod mit COVID-19.

Auch nach Bereinigung um die Anzahl der "geschützten Personen", d. h. die Anzahl der Personen, die in der Gruppe mit Maskenpflicht im Vergleich zur Gruppe ohne Maske nicht infiziert waren, blieb das relative Risiko mit 1,52 [1,24-1,72] signifikant hoch. Durch die Analyse der Übersterblichkeit in Kansas stellt diese Studie fest, dass über $95 \%$ dieses Effekts allein auf COVID-19 zurückzuführen sind. Die Ursache für diesen Trend und den möglichen Zusammenhang zwischen den Langzeitfolgen von SARS-CoV-2 und Gesichtsmasken wird in dieser Studie durch die Theorie des "Fögen-Effekts" erklärt. Dieser beschreibt die tiefe Re-Inhalation von rei- 
nen Virionen, die als Tröpfchen in den Gesichtsmasken hängen geblieben sind, was die Prognose verschlechtern kann.

Diese Ergebnisse deuten darauf hin, dass die Verwendung von Gesichtsmasken bei der COVID19-Pandemie entgegen ihrem Zweck zu einer Erhöhung der Todesrate beigetragen hat, was die Maskenpflicht zu einer höchst fragwürdigen epidemiologischen Intervention macht.

\section{Manuscript: Preprint V3 (Final)}

Note: This final preprint version has undergone professional scientific editing.

\section{Hosted file}

Facemasks_and_COVID-19_case_fatality_rate_PREPRINT_V3.pdf available at https://authorea.com/ users/391921/articles/505927-facemasks-and-covid-19-case-fatality-rate

\section{Supplementary Appendix:}

\section{Hosted file}

Supplementary Appendix.pdf available at https://authorea.com/users/391921/articles/505927facemasks-and-covid-19-case-fatality-rate

Data and calculations sheet: 


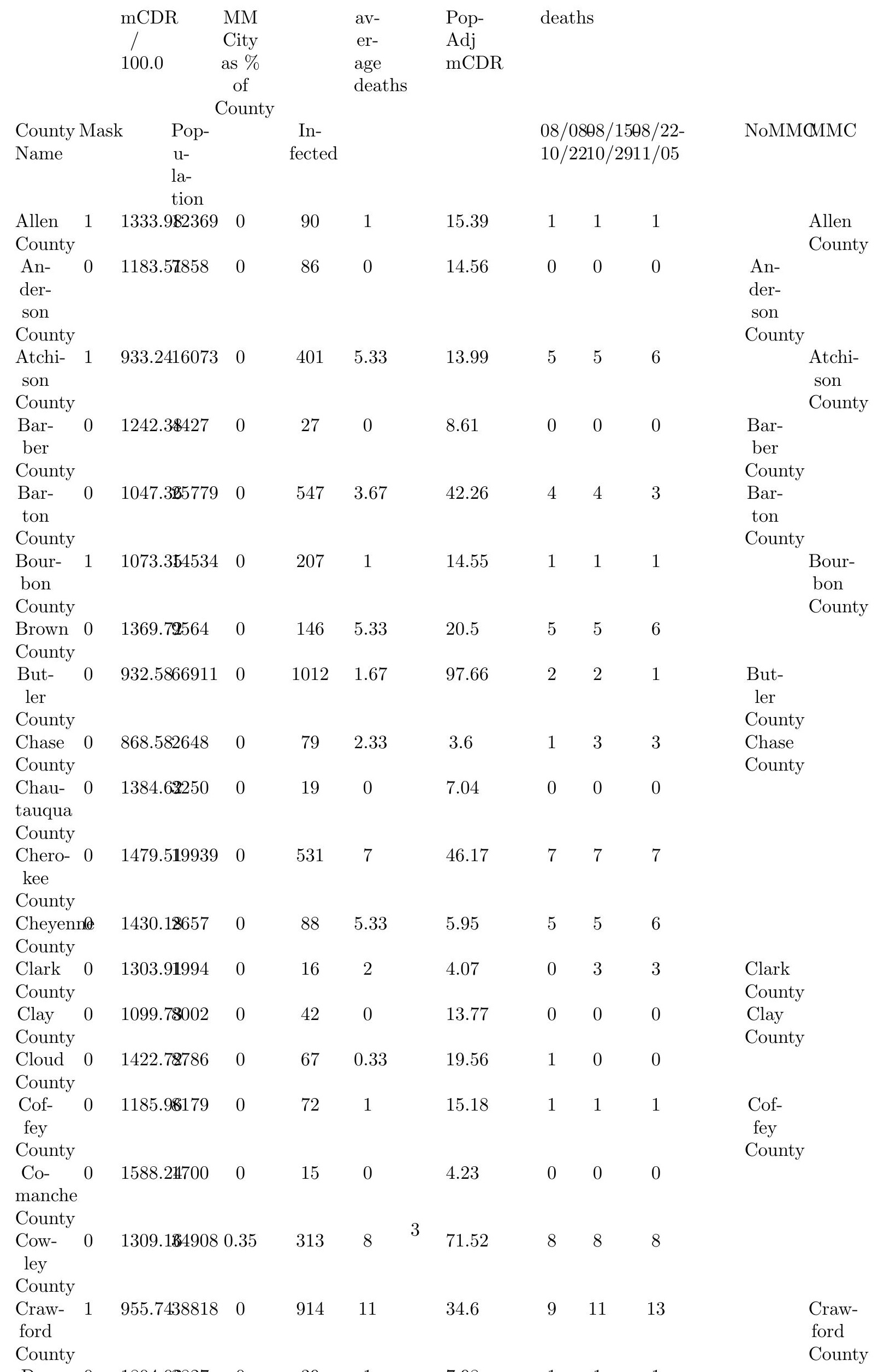

